\title{
Mesh Infection and Migration after Umbilical Hernia Repair
}

\author{
Valter Ripetti", Vincenzo La Vaccara, Eleonora Angelini, Giovan Battista Giorgio, Rossana Alloni \\ Department of General Surgery, Campus Bio-Medico University, Rome, Italy. \\ Email: *v.ripetti@unicampus.it, v.lavaccara@unicampus.it, e.angelini@unicampus.it, g.giorgio@unicampus.it, r.alloni@unicampus.it
}

Received August 16, 2013; revised September 14, 2013; accepted September 21, 2013

Copyright (c) 2013 Valter Ripetti et al. This is an open access article distributed under the Creative Commons Attribution License, which permits unrestricted use, distribution, and reproduction in any medium, provided the original work is properly cited.

\begin{abstract}
Mesh infection and migration are dangerous and common complications after hernia repair. Many factors cause mesh migration, which may or may not be associated to infection. Before performing hernia repair using a mesh, it is important to choose the right device to avoid this kind of complication, above all in the presence of contaminated wounds or fields. We describe two cases of mesh infection and migration after umbilical hernia repair which were treated, in accordance with recommendations in the literature, by removing the infected mesh and replacing it with a biological mesh. Our experience confirms the feasibility of using biological mesh to perform umbilical hernia repair after infection, with consequent migration, of the previously placed mesh. Our cases are the first to be reported in which mesh migration is associated with infection after umbilical hernia repair, and they demonstrate the advantages of biologic implants in abdominal wall reconstruction.
\end{abstract}

Keywords: Umbilical; Infection; Migration Hernia; Mesh

\section{Introduction}

Migration of a surgical mesh, and consequent infection, are dangerous and common complications in patients who have undergone hernia repair. Many reports of plug and mesh migration or dislocation after open or laparoscopic inguinal hernia repair can be found in literature. This kind of complication can cause recurrence of inguinal hernia, intestinal occlusion, chronic neuralgia, and intestinal erosions, and thus peritonitis and enteric fistula [1].

In literature, cases of mesh migration after umbilical hernia repair are extremely rare. There is only one paper, by Di Muria et al., describing a case of polypropylene mesh migrating and completely penetrating an ileal loop, with adhesion to the abdominal wall. Resection of the ileal loop together with the surrounding abdominal wall was performed, and the parietal defect was repaired with single polypropylene stitches, avoiding the use of prosthetic material [2]. We describe 2 cases regarding mesh migration and infection after umbilical hernia repair, and provide a review of the literature.

\section{Case Presentation 1}

The patient was a 60-year-old female whose medical

${ }^{*}$ Corresponding author. history included autoimmune thyroditis and hypertension. She was a smoker, and her BMI was 30 .

The patient underwent direct umbilical hernia repair at the age of 36 years, and 20 years later developed a recurrence which was treated by intra-peritonium repair using a Composite ${ }^{\circledR}$ mesh (Bard Inc., USA).

After 4 more years she was admitted to another hospital for recurrent fever and abdominal swelling. Abdominal X-ray was negative. A subsequent abdominal CT scan demonstrated the presence of fluid collection with gas bubbles near the mesh. Both fever and local swelling disappeared in response to non-specific antibiotic therapy, and she was discharged after 10 days. Four months later, she presented with periumbilical swelling with presence of cutaneous fistula, so a new CT scan was performed. This revealed persistence of fluid collection with gas bubbles near the mesh (Figure 1).

Bacterial culture was positive for Streptococcus constellatus. The patient was therefore admitted to our department and underwent surgical treatment. Intraoperative findings revealed the presence of a subcutaneous purulent collection and migration of the mesh into an ileal loop (Figure 2). An intraoperative sampling confirmed the growth of Streptococcus constellatus. After removing the prosthesis en bloc with surrounding skin structures, we first performed resection of the involved 


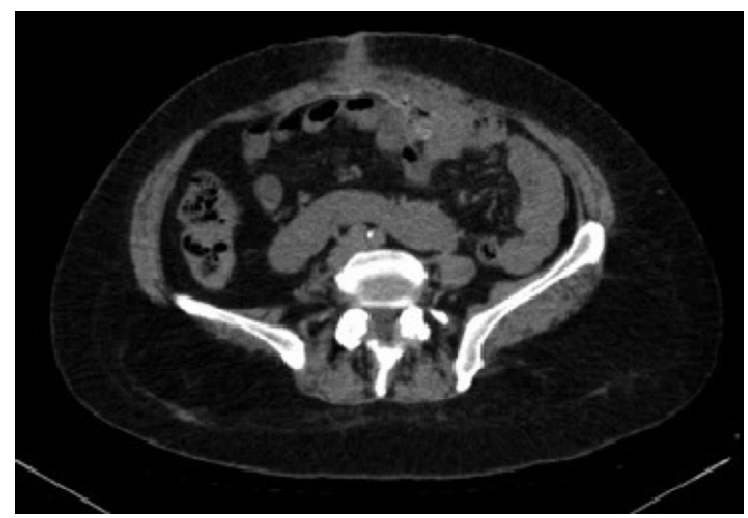

Figure 1. Fluid collection with gas bubbles near the mesh.

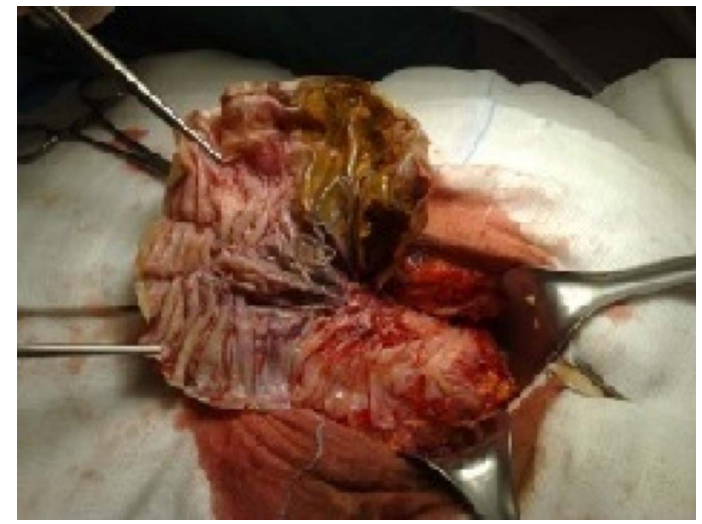

Figure 2. Prosthesis penetrated in ileal loop.

ileal loop. After primary suture, repair of the posterior rectus sheath was performed using vicryl 2, and an overlay mesh of Permacol ${ }^{\mathrm{TM}}(10 \times 15 \mathrm{~cm})$ was secured with a series of sutures (2/0 prolene; ethicon). The external rectus sheath was re-approximated at midline with a series of 2 vicryl sutures. Additionally, a JacksonPratt drain was placed in the subcutaneous plane, and was left in place for four days. The patient was treated with antibiotic therapy (Cefazolina Teva) until her discharge. The postoperative course was uneventful and the patient was discharged on postoperative day 6 . The patient was also instructed to wear an abdominal binder for at least two months. At 24 months follow-up, there was no evidence of recurrence or other symptoms.

\section{Case Presentation 2}

A 56-year-old female, also a smoker, who had a history of umbilical hernia repair with a Goretex ${ }^{\circledR}$ (Japan GoreTex, Inc., Japan) mesh six years previously, was admitted to our department with pus output from a periumbilical swelling, and external mesh migration (Figure 3). BMI was 31. Bacterial culture, done before admission, was positive for Escherichia coli, Streptococcus agalactiae and Streptococcus dysgalactiae.

The patient underwent surgical treatment, and intraop-

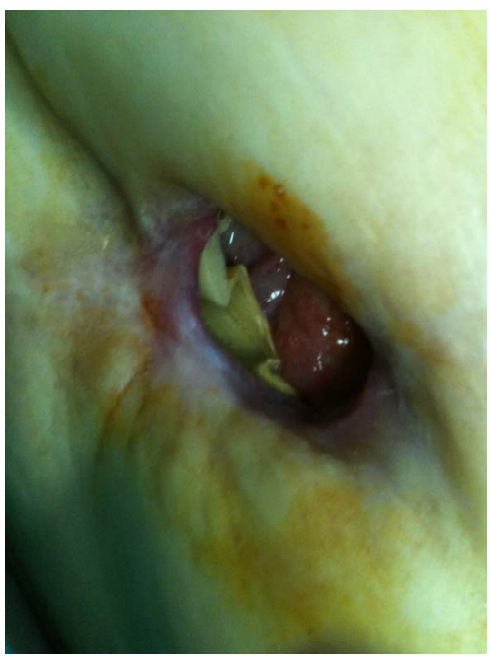

Figure 3. External mesh migration.

erative findings revealed the presence of a subcutaneous purulent collection confirming the multiple infections mentioned above. We performed complete removal of the mesh, together with surrounding skin structures, and an underlay abdominal wall repair using a mesh of Permacol $^{\mathrm{TM}}(10 \times 15 \mathrm{~cm})$ which was secured with a series of sutures (2/0 prolene; ethicon) to the posterior rectus sheath. Primary suture repair of the posterior and external rectus sheath was performed using vicryl 2 . Additionally, a tubular suction drain was placed in the subcutaneous plane. the drain was left in place for two days until it expressed less than $50 \mathrm{ml} / 24 \mathrm{~h}$. The patient was treated with antibiotic therapy (Cefazolina Teva) until her discharge. The postoperative course was uneventful and the patient was discharged on postoperative day 3. The patient was also instructed to wear an abdominal binder for at least two months. At 24 months follow-up, there was no evidence of recurrence or other symptoms.

\section{Discussion}

Umbilical hernia is relatively common in adults, but accounts for only $3 \%-8.5 \%$ of abdominal hernias, third in incidence after inguinal (70\% - 75\%) and femoral (6\% $17 \%$ ) hernias [3]. Meshes have significantly reduced the incidence of recurrence after umbilical hernia repair, from $54 \%$ to less than $10 \%$ [4]. On the other hand, Arroyo et al. did not report a significant relationship between recurrence rate and size of the hernia, comparing suture and mesh repair of umbilical defects in a randomized clinical trial [5]. Although open primary repair still remains the operation of choice for some surgeons faced with defects of less than $2 \mathrm{~cm}$ in diameter [6], others prefer the use of a mesh plug for defects of up to $3 \mathrm{~cm}$ in diameter, and a mesh sheet for larger lesions [5].

Regardless of the technique used, the risk of mesh in- 
fection remains a serious problem. The incidence of mesh infection in open surgery $(6 \%-10 \%)[7,8]$ drops to $0 \%-3.6 \%$ with laparoscopic procedure [9], thanks to mesh introduction through trocars, which avoids skin contact, and placement far from the trocar incisions [10]. In literature, most prosthetic infections are reported to be due to skin pathogens [11], although in our experience the pathogens involved were not part of the cutaneous flora.

In 2001, an experts' meeting about the classification and treatment of incisional hernia reported a higher mesh removal rate after infection in laparoscopic repair $(0 \%$ $7 \%)$ than after open repair (0\% - 4\%) [12]. This was probably due to a more prevalent use of ePTFE mesh in laparoscopic repair: this type of mesh produces less bowel adhesion than polypropylene or polyester mesh but, once infected, must more often be removed [13]. In fact, infection of polypropylene mesh can be managed locally with surgical drainage and excision of exposed unincorporated segments, whereas infection of ePTFE mesh requires removal of the prosthetic material because its microporous $(10 \mu \mathrm{m})$ surface allows fluid retention and bacterial contamination, but not leukocyte diapedesis [8]. The removal of infected ePTFE mesh becomes necessary because of its intraperitoneal placement, in contact with the intestinal loops, which leads to the involvement of adjacent organs.

According to a meta-analysis of cohort studies based on 2418 patients, published in 2011 by Mavros et al., our patients had risk factors significantly associated with the development of mesh infection after hernioplasty, such as high BMI score and tobacco smoking [14]. In our two cases, mesh migration was caused by infection of the mesh, as suggested by Agrawal and Avill [15], who hypothesized two possible mechanisms of mesh migration. In the first of these, displacement of the mesh occurs along a path of low resistance resulting either from inadequate fixation or from external displacing forces. In the second, referred to as secondary migration, the mesh moves gradually and slowly through the anatomic planes due to erosion induced by foreign body reaction; the migration depends on the nature of the mesh biomaterial and the type of fixation of the mesh, if fixed. On the other hand, Chowbey et al. suggested that the cut edges of the mesh become sharp, damaging the surface of the viscus and evoking an inflammatory reaction, thereby leading to weakness, erosion, and finally infection [16].

Because of a high morbidity rate due to mesh explantation after mesh infection (hernia recurrence, damage to surrounding structures, loss of domain, risk of enterotomy or enterocutaneous fistula formation), it is essential to observe rigorous asepsis during surgical placement of an appropriate mesh, to avoid the formation of intraparietal haematomas which could cause infection and, in the end, mesh explantation [13].

At the 30th International Congress of the European Hernia Society in 2009, different properties of the prosthesis (such as pore size, geometry, active surface area, memory, affinity for water, elasticity, and polymer type) were taken into account to identify an "ideal" mesh prosthesis. The pore size of meshes is crucial in defining the surgical activity profile, which includes handling, flexibility, memory, fibrosis, and foreign body reaction. Although lightweight macroporous meshes may lead to a higher risk of adhesions, the panel agreed that they seem to be safer to use in a contaminated environment because of their greater flexibility and lower risk of foreign body reaction and fibrosis [17].

Recently, greater use of biocompatible implants, such as Permacol ${ }^{\mathrm{TM}}$, has been necessary, because of complications with infection, and adhesions associated with widespread use of prosthetic materials, such as polypropylene mesh and ePTFE, to reconstruct abdominal wall defects. Permacol $^{\mathrm{TM}}$ (acellular porcine dermis) is used as a dermal scaffold, vascularised and remodelled to reconstruct the abdominal wall, particularly in the presence of contaminated or potentially contaminated wounds [18]. Thanks to its structure, which is similar to human dermis, Permacol $^{\mathrm{TM}}$ is considered to be equally non-allergenic, nontoxic, and devoid of foreign body reaction. Additional collagen crosslinking, which allows fibroblast infiltration and neovascularisation, incorporating the implant into the surrounding host tissue [19], confers greater stability, decreasing degradation by collagenase and resulting in a more long-term prosthesis [20].

Literature report conflicting results with regard to Permacol's ability to become vascularized and incorporated into host tissue [20-23], showing conflicting data on how chemical crosslinking influences vascular incorporation. However, a recent report demonstrated clinical and histological evidence of vascular incorporation in a full-thickness [24]. Regardless of whether or not chemical crosslinking may lead to integration of the mesh and decreased ability for vascularization, this is offset by its resistance to degradation and its long-term strength. In fact it was demonstrated the chemical crosslinking of collagen in Permacol ${ }^{\mathrm{TM}}$ confers more long-term stability and strength [25].

According to a report on histologic and biomechanical evaluation of crosslinked and non-crosslinked biologic meshes in a porcine model of ventral incisional hernia repair, Permacol ${ }^{\mathrm{TM}}$ showed increased cellular infiltration between 1 and 6 months, with some scores to the noncrosslinked materials at 6 months, and persistence of these scores at 12 months. However, because all crosslinked and non-crosslinked biologic meshes showed equivalent cellular infiltration at later time points, it is possible that crosslinking might not influence cellular 
infiltration substantially in the long term. In general, the crosslinked materials also demonstrated increased scores at 12 months for cell types, ECM deposition, scaffold degradation, fibrous encapsulation, and neovascularization compared with their scores at 1 month. Although many differences were identified among the biologic meshes examined in this study, it is difficult to say whether any of these biologic meshes are more biocompatible than the others for ventral hernia repair because there are such wide variations in both the clinical scenarios analyzed and the biological responses of individual patients [26]. Also, a retrospective comparative study, in 2012, on synthetic and biological meshes in component separation for abdominal wall hernia repairs, showed a recurrence rate in Permacol ${ }^{\mathrm{TM}}$ repair in contaminated fields of $7.7 \%$, compared with Alloderm (19\%), synthetic mesh (25.6\%), and component separation (12\%). This review suggests that the crosslinked mesh, Permacol ${ }^{\mathrm{TM}}$, has the lowest failure rate and the longest time to failure, particularly in contaminated or infected fields [27]. In our cases there was a late clinical presentation of mesh migration, where pus output was the only sign of infection, with none of the abdominal pain or bowel occlusion reported by Di Muria [2]. In the first case the periumbilical pus output was caused by enterocutaneous fistula formation, and in the second it was due to partial external mesh migration. In both cases we decided, in accordance with the literature concerning mesh infection, to remove the infected mesh and perform an abdominal wall repair using bioprosthesis mesh to ensure lower risk of recurrence and infection. Our experience confirms this biodegradable matrix as a safe and useful tool; therefore it should be the device of choice when there is high risk of infection and migration, or of major complications such as intestinal perforation. In conclusion, our cases are the first to be reported in which mesh migration is associated with infection after umbilical hernia repair, and they demonstrate the advantages of biologic implants in abdominal wall reconstruction. Biological devices such as Permacol ${ }^{\mathrm{TM}}$ should be considered in cases of contaminated wounds, immunesuppressed patients, and previously placed, infected mesh, even if high cost limits their routine use.

\section{REFERENCES}

[1] M. Basoglu, M. I. Yildirgan, I. Yilmaz, et al., "Late Complications of Incisional Hernias Following Prosthetic Mesh Repair,” Acta Chirurgica Belgica, Vol. 104, No. 4, 2004, pp. 425-428.

[2] A. Di Muria, V. Formisano, F. Di Carlo, et al., "Small Bowel Obstruction by Mesh Migration after Umbilical Hernia Repair,” Annali Italiani di Chirurgia, Vol. 78, No. 1, 2007, pp. 59-60.

[3] N. Dabbas, K. Adams, K. Pearson, et al., "Frequency of
Abdominal Wall Hernias: Is Classical Teaching Out of Date?” JRSM Short Reports, Vol. 2, No. 1, 2007, p. 5.

[4] D. S. Edelman and C. F. Bellows, "Umbilical Herniorraphy Reinforced with Biologic Mesh," The American Surgery, Vol. 76, No. 11, 2010, pp. 1205-1209.

[5] A. Arroyo, P. García, F. Pérez, et al., "Randomized Clinical Trial Comparing Suture and Mesh Repair of Umbilical Hernia in Adults,” British Journal of Surgery, Vol. 88, No. 10, 2001, pp. 1321-1323. http://dx.doi.org/10.1046/j.0007-1323.2001.01893.x

[6] B. E. Wright, J. Beckerman, M. Cohen, et al., "Is Laparoscopic Umbilical Hernia Repair with Mesh a Reasonable Alternative to Conventional Repair?” The American Journal of Surgery, Vol. 184, No. 6, 2002, pp. 505-508. http://dx.doi.org/10.1016/S0002-9610(02)01071-1

[7] W. S. Cobb, A. M. Carbonell, C. L. Kalbaugh, et al., "Infection Risk of Open Placement of Intraperitoneal Composite Mesh,” The American Surgery, Vol. 75, No. 9, 2009, pp. 762-768.

[8] S. Petersen, G. Henke, M. Freitag, et al., "Deep Prosthesis Infection in Incisional Hernia Repair: Predictive Factors and Clinical Outcome,” European Journal of Surgery, Vol. 167, No. 6, 2001, pp. 453-457. http://dx.doi.org/10.1080/110241501750243815

[9] J. R. Eriksen, T. Gogenur and J. Rosenberg, "Choice of Mesh for Laparoscopic Ventral Hernia Repair,” Hernia, Vol. 11, No. 6, 2007, pp. 481-492. http://dx.doi.org/10.1007/s10029-007-0282-8

[10] D. Foschi, F. Corsi, P. Cellerino, et al., "Late Rejection of the Mesh after Laparoscopic Hernia Repair,” Surgical Endoscopy, Vol. 12, No. 5, 1998, pp. 455-457. http://dx.doi.org/10.1007/s004649900704

[11] W. S. Cobb, K. W. Kercher and B. T. Heniford, "Laparoscopic Repair of Incisional Hernias," Surgical Clinics of North America, Vol. 85, No. 1, 2005, pp. 91-103. http://dx.doi.org/10.1016/j.suc.2004.09.006

[12] M. Korenkov, A. Paul, S. Sauerland, et al., "Classification and Surgical Treatment of Incisional Hernia: Results of an Experts' Meeting," Langenbeck's Archives of Surgery, Vol. 386, No. 1, 2001, pp. 65-73. http://dx.doi.org/10.1007/s004230000182

[13] V. M. Sanchez, Y. E. Abi-Haidar and K. M. Itani, "Mesh Infection in Ventral Incisional Hernia Repair: Incidence, Contributing Factors, and Treatment," Surgical Infections (Larchmt), Vol. 12, No. 3, 2011, pp. 205-210. http://dx.doi.org/10.1089/sur.2011.033

[14] M. N. Mavros, S. Athanasiou, V. G. Alexiou, et al., "Risk Factors for Mesh-Related Infections After Hernia Repair Surgery: A Meta-Analysis of Cohort Studies," World Journal of Surgery, Vol. 35, No. 11, 2011, pp. 2389-2398. http://dx.doi.org/10.1007/s00268-011-1266-5

[15] A. Agrawal and R. Avill, "Mesh Migration Following Repair of Inguinal Hernia: A Case Report and Review of Literature,” Hernia, Vol. 10, No. 1, 2006, pp. 79-82. http://dx.doi.org/10.1007/s10029-005-0024-8

[16] P. K. Chowbey, N. Bagchi, A. Goel, et al., ”Mesh Migration into the Bladder after TEP Repair: A Rare Case Report," Surgical Laparoscopy, Endoscopy \& Percutaneous Techniques, Vol. 16, No. 1, 2006, pp. 52-53. 
http://dx.doi.org/10.1097/01.sle.0000202185.34666.f1

[17] S. Bringman, J. Conze, D. Cuccurullo, et al., "Hernia Repair: The Search for Ideal Meshes,” Hernia, Vol. 14, No. 1, 2010, pp. 81-87. http://dx.doi.org/10.1007/s10029-009-0587-x

[18] P. W. Hsu, C. J. Salgado, K. Kent, et al., ”Evaluation of Porcine Dermal Collagen (Permacol) Used in Abdominal Wall Reconstruction," Journal of Plastic, Reconstructive \& Aesthetic Surgery, Vol. 62, No. 11, 2009, pp. 14841489. http://dx.doi.org/10.1016/j.bjps.2008.04.060

[19] C. Harper, "Permacol: Clinical Experience with a New Biomaterial,” Hospital Medicine, Vol. 62, No. 2, 2001, pp. 90-95.

[20] M. L. Jarman-Smith, T. Bodamyali, C. Stevens, et al., "Porcine Collagen Crosslinking, Degradation, and Its Capability for Fibroblast Adhesion and Proliferation," Journal of Materials Science: Materials in Medicine, Vol. 15, No. 8, 2004, pp. 925-932. http://dx.doi.org/10.1023/B:JMSM.0000036281.47596.cC

[21] F. Zheng, Y. Lin, E. Verbeken, et al., "Host Response after Reconstruction of Abdominal Wall Defects with Porcine Dermal Collagen in a Rat Model," American Journal of Obstetrics \& Gynecology, Vol. 191, 2004, pp. 1961-1970.

[22] T. M. Macleod, G. Williams, R. Sanders, et al., "Histological Evaluation of Permacol as a Subcutaneous Im- plant over a 20-Week Period in the Rat Model,” British Journal of Plastic Surgery, Vol. 58, No. 4, 2005, pp. 518532.

[23] J. A. O’Brien, R. Ignotz, R. Montilla, et al., “Long-Term Histologic and Mechanical Results of a Permacol Abdominal Wall Explant," Hernia, Vol. 15, No. 2, 2011, pp. 211-215. http://dx.doi.org/10.1007/s10029-010-0628-5

[24] T. S. Satterwhite, S. Miri, et al., "Abdominal Wall Reconstruction with Dual Layer Crosslinked Porcine Dermal Xenograft: The “Pork Sandwich” Herniorraphy,” Journal of Plastic, Reconstructive \& Aesthetic Surgery, Vol. 65, No. 3, 2012, pp. 333-341. http://dx.doi.org/10.1016/j.bjps.2011.09.044

[25] K. E. Mulier, A. H. Nguyen, J. P. Delaney, et al., "Comparison of Permacol ${ }^{\mathrm{TM}}$ and Strattice ${ }^{\mathrm{TM}}$ for the Repair of Abdominal Wall Defects," Hernia, Vol. 15, 2011, pp. 315-319.

[26] C. R. Deeken, L. Melman, et al., "Histologic and Biomechanical Evaluation of Crosslinked and Non-Crosslinked Biologic Meshes in a Porcine Model of Ventral Incisional Hernia Repair," Journal of the American College of Surgeons, Vol. 212, No. 5, 2011, pp. 880-888.

[27] N. J. Smart, M. Marshall and I. R. Daniels, "Biological Meshes: A Review of Their Use in Abdominal Wall Hernia Repairs,” Surgeon, Vol. 10, No. 3, 2012, pp. 159-171. 